\title{
Das maligne Melanom der Nasenschleimhaut
}

\section{Zusammenfassung}

Das maligne Melanom der Schleimhäute ist auch im Bereich der Nasen- und Nasennebenhöhlen ein selten vorkommender Tumor, der sich durch eine Epistaxis oder durch die Zeichen einer nasalen Obstruktion zu erkennen gibt. Da die klinische Symptomatik in der Regel erst bei einem fortgeschrittenen Tumorwachstum beobachtet wird, muss die Prognose der nasalen Schleimhautmelanome als ungünstig angesehen werden. Das in der folgenden Kasuistik beschriebene Septum-Melanom entspricht in typischer Weise dem klinischen Verlauf nasaler Schleimhautmelanome. Die Diagnose war infolge anhaltender Nasenblutungen gestellt worden, wobei zu diesem Zeitpunkt bereits eine intrazerebrale Metastasierung nachgewiesen werden konnte.

\section{Einleitung}

Obwohl die Schleimhautmelanome im Nasen- und Nasennebenhöhlenbereich zweifellos selten sind, finden sich in der Literatur teilweise deutlich abweichende Angaben zu ihrer Häufigkeit. Diese unterschiedliche Datenlage ist darauf zurückzuführen, dass Patienten mit nasalen Schleimhautmelanomen durch verschiedene Fachrichtungen betreut werden. Auf Grund der speziellen Lokalisation wird die Mehrzahl der betroffenen Patienten in den HNO-Kliniken behandelt, während ein nasales Schleimhautmelanom in der Dermatologie als Rarität gewertet werden muss. Darüber hinaus sind die fachspezifischen Auswertungskriterien, auf denen die Häufigkeitsangaben basieren, unterschiedlich und nicht immer miteinander vergleichbar. Von wenigen Ausnahmen abgesehen wird in den dermatologischen Studien die Anzahl der Schleimhautmelanome unabhängig von ihrer Lokalisation im Vergleich zur Gesamtzahl aller malignen Melanome betrachtet, während in der Hals-Nasen-Ohrenheilkunde alle hier beobachteten Schleimhautmelanome als prozentualer Anteil der malignen Melanome im Kopf-Hals-Bereich ausgewiesen werden. Betrachtet man die Gesamtheit aller malignen Melanome unter dem Aspekt der Lokalisation ihres Auftretens, ergibt sich auf der Grundlage dermatologischer Untersuchungen eine Häufigkeit maligner Melanome im Kopf-Hals-Bereich von $9,8-15,1 \%$, ohne dass jedoch der Anteil der in diesem Bereich auftretenden Schleimhautmelanome gesondert ausgewiesen wurde $[1,2]$. Im Gegensatz hierzu finden sich in der HNO-Literatur vereinzelt Studien, bei denen der prozentuale Anteil der Schleimhautmelanome im Kopf-Hals-Bereich erfasst worden ist. Demzufolge werden 3,2-8,9\% aller Kopf- und Halsmelanome

Institutsangaben

Hautklinik, Klinikum Bremerhaven Reinkenheide (Chefarzt: Dr. G. Wagner)

Korrespondenzadresse

Dr. Gunnar Wagner · Hautklinik · Klinikum Bremerhaven Reinkenheide · Postbrookstraße 103 .

27574 Bremerhaven

Bibliografie

Akt Dermatol 2006; 32: 256-259 @ Georg Thieme Verlag KG Stuttgart · New York

DOI 10.1055/s-2006-925043 · ISSN 0340-2541 
im Bereich der Schleimhäute diagnostiziert $[3,4]$. In der uns zur Verfügung stehenden Literatur fanden sich nur zwei Untersuchungen, bei denen die Häufigkeit der Schleimhautmelanome im Nasen- und Nasennebenhöhlenbereich als Teilmanifestation der am gesamten Integument aufgetretenen malignen Melanome erfasst worden ist. Bei diesen Untersuchungen lag die Häufigkeit nasaler Schleimhautmelanome bei 0,6 und $0,7 \%$ [5,6]. Im eigenen Krankengut der Hautklinik Bremerhaven waren von 485 diagnostizierten malignen Melanomen 40 im Kopf-Hals-Bereich lokalisiert, entsprechend einer Häufigkeit von 8,2\%. Nur der hier vorgestellte Patient wies dabei eine nasale Schleimhautmanifestation auf. Damit beträgt der Anteil der Schleimhautmelanome bei unseren Patienten 2,2\% der malignen Melanome im KopfHals-Bereich und nur 0,2\% aller in unserer Klinik diagnostizierten malignen Melanome. Auch im Zentralregister Malignes Melanom der Deutschen Dermatologischen Gesellschaft findet sich für Schleimhautmelanome eine vergleichbare Größenordnung. Von 70901 dort bisher erfassten malignen Melanomen waren $278 \mathrm{im}$ Bereich aller Schleimhautregionen lokalisiert, was einer Häufigkeit von 0,4\% entspricht [2]. Beim Vergleich der vorliegenden Daten lässt sich somit zusammenfassend feststellen, dass Schleimhautmelanome auch im HNO-Bereich insgesamt selten sind, offensichtlich aber doch häufiger vorkommen, als man dies aus dermatologischer Sicht vermuten würde.

\section{Kasuistik}

\section{Anamnese}

Der hier vorgestellte 34-jährige Patient war mit der Diagnose eines beidseitigen Septumhämangioms in die HNO-Klinik des Hauses eingewiesen worden. Er gab an, seit 4-5 Wochen fast täglich Nasenblutungen bemerkt zu haben. Mit einer posttraumatischen Epistaxis hatte er sich 6 Monate zuvor bei einem HNO-Arzt vorgestellt. Der bereits damals gesehene Tumor war auch zu diesem Zeitpunkt als Septumhämangiom eingeordnet worden.

\section{Dermatologischer Befund}

Im knorpeligen Anteil des linken Nasenseptums ein nur teilweise einsehbarer, scharf begrenzter, bläulich-schwarz pigmentierter Tumor mit oberflächlichen Erosionen und hämorrhagischen Krusten (Abb.1). Am rechten Nasenseptum kleinerer Tumoranteil gleicher Morphologie.

\section{Histopathologischer Befund}

Plattenepithel der Nasenschleimhaut mit darunter liegender diffuser Infiltration durch spindelige und epitheloide melanozytäre Tumorzellen. Deutlich verschobene Kernplasmarelation, nukleäre Hyperchromasie, Pleomorphie und prominente Nukleolen. Dichtes Melanophageninfiltrat. Immunhistochemisch positive Reaktivität für S-100 und Melan-A, negative Befunde bei Pan-Cytokeratin, EMA, CD-3, CD-20 und CD-30.

Diagnose: Malignes Melanom der Nasenschleimhaut

\section{Ergänzende Untersuchungen}

MRT Gesichtsschädel/Hirnschädel: Tumoröse Raumforderung am Nasenseptum mit Destruktion der Siebbeinplatte und intrakraniellen Anteilen. Zweite Läsion im Lobus frontalis inferior mit enger topografischer Beziehung zum Circulus Willisii.

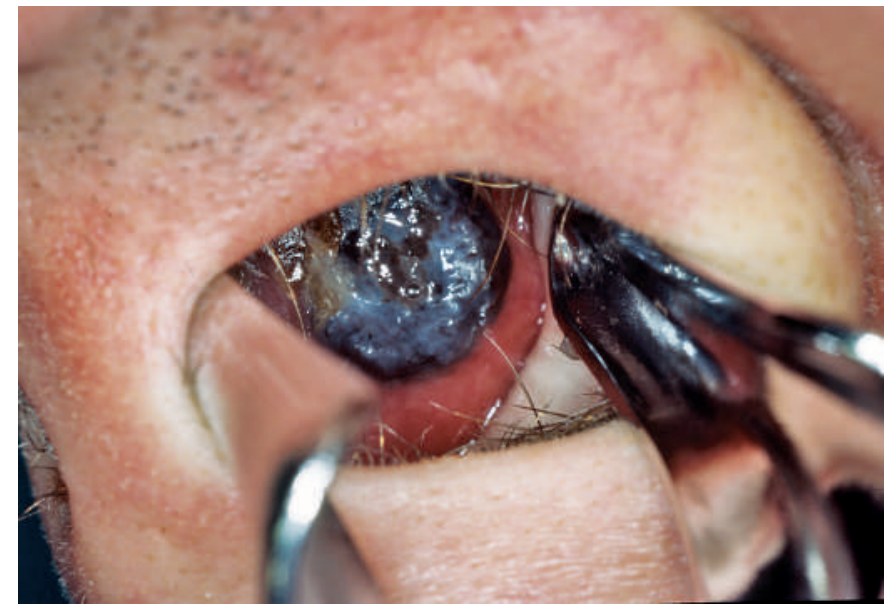

Abb. 1 Knotiges, an der Oberfläche erodiertes Schleimhautmelanom Nasenseptum links.

CT Hals, Thorax und Abdomen, Rö.-Thorax, Oberbauchsonographie und zervikale Sonographie ohne Nachweis metastasentypischer Befunde.

Routinemäßige Laboruntersuchungen einschließlich LDH und S-100 ohne pathologische Befunde.

\section{Therapie und Verlauf}

Nach bioptischer Sicherung der Diagnose erfolgte die operative Entfernung des Tumors durch einen gemeinsamen Eingriff der Kollegen der HNO-Klinik und der Neurochirurgie. Dabei wurden zunächst das Septum und die Nasenmuscheln exzidiert (Abb. 2 u.3). Durch eine anschließende bifrontale Kraniotomie ließ sich das Tumorwachstum im Bereich der linken Siebbeinplatte, der Dura mater und des Frontalhirnes weiter verfolgen. Die betroffenen Gewebestrukturen wurden operativ entfernt, wobei die spätere histopathologische Aufarbeitung zeigte, dass eine R0-Resektion nicht erreicht worden war. Daraufhin erfolgte eine Bestrahlung des Gesamthirns mit einer kumulativen Dosis von 40 Gy. Gleichzeitig wurde eine Chemotherapie mit Temozolamid eingeleitet. Bei insgesamt schlechter Prognose bleibt der weitere Verlauf abzuwarten.

\section{Diskussion}

Die Schleimhautmelanome im Kopf-Hals-Bereich zeigen eine unterschiedliche topografische Verteilung, wobei ihre Häufigkeit im Bereich der Nasen- und Nasennebenhöhlen mit 34-60\% angegeben wird, gefolgt von der Mundhöhle mit 27-50\% und der Pharynx-Larynx-Region mit 8-15\% [4,7]. In der Mehrzahl der Fälle sind die auskleidenden Schleimhäute der genannten Organsysteme betroffen, während Schleimhautmelanome im Bereich anderer Strukturen, z. B. an der Zunge oder an den Tonsillen, nur sehr vereinzelt beobachtet wurden [8-10]. Auch bei einem direkten Vergleich der Tumorhäufigkeit zwischen den Nasenhöhlen und den Nasennebenhöhlen findet sich eine ungleiche Verteilung der Schleimhautmelanome. In einer Gruppe von 56 Patienten fanden Freedman et al. 29 Schleimhautmelanome 


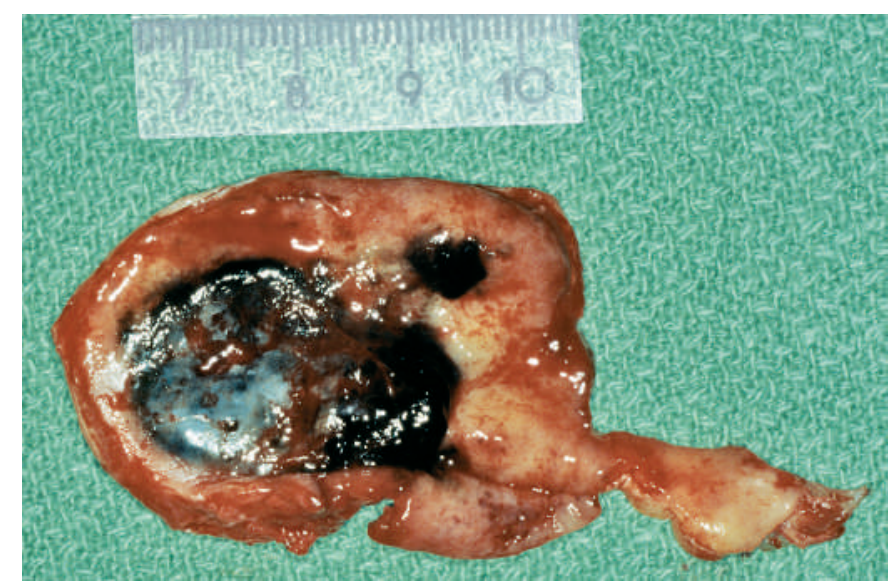

Abb. 2 Op-Präparat mit ausgedehnten Melanomanteilen Septum links.

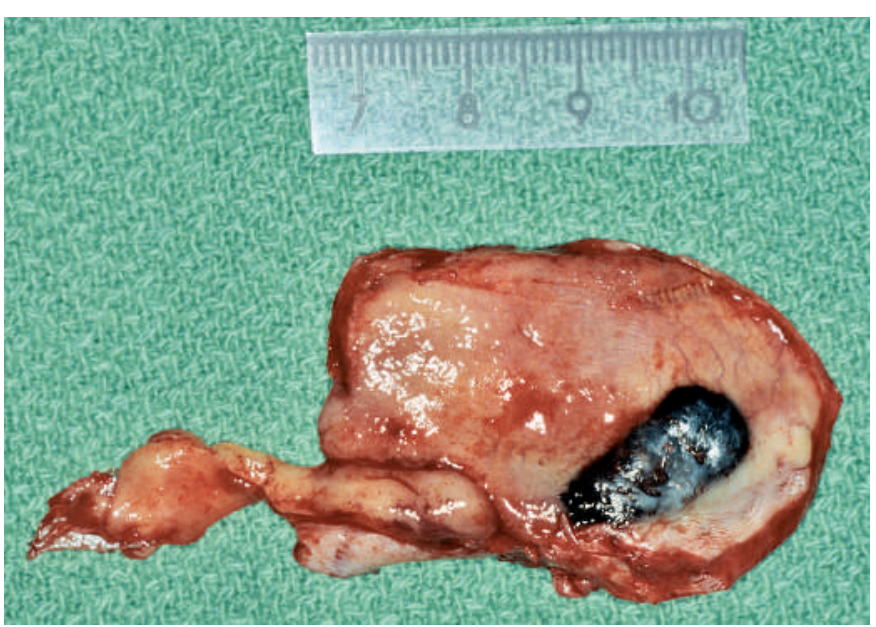

Abb. 3 Op-Präparat mit Befund am Septum rechts.

im Bereich der Nasenhöhlen (51,8\%) und 18 in den Nasennebenhöhlen $(32,1 \%)$ lokalisiert. Bei den verbliebenen 9 Patienten $(16,1 \%)$ war eine topografische Zuordnung der primären Lokalisation auf Grund der Tumorausdehnung zum Zeitpunkt der Diagnose nicht mehr möglich gewesen [11]. In einer weiteren Untersuchung von Holdcraft und Gallagher, bei der die Daten von 39 Patienten ausgewertet werden konnten, hatten sich 21 Schleimhautmelanome in den Nasenhöhlen $(53,8 \%)$ und 7 in den Nasennebenhöhlen (18,0\%) entwickelt. Auch bei dieser Untersuchung konnte die ursprüngliche Lokalisation der übrigen 11 Schleimhautmelanome $(28,0 \%)$ nicht mehr festgestellt werden, da sowohl die Nasen- als auch die Nasennebenhöhlen von Tumormassen ausgefüllt waren [12]. Deutlich voneinander abweichende Angaben finden sich in der Literatur zur Häufigkeit der Tumorlokalisation innerhalb der Nasenhöhlen. Freeman et al. beobachteten die meisten Schleimhautmelanome am Nasenseptum, während die laterale Nasenwand und die Nasenmuscheln deutlich seltener als Ausgangspunkt der Tumorentwicklung identifiziert werden konnten [11]. Bei den Untersuchungen von Nandapalan et al. waren hingegen am häufigsten die mittlere Nasenmuschel und der Übergang zu den Nasennebenhöhlen betroffen gewesen, deutlich seltener die untere Nasenmuschel und das Septum [13]. Die Schleimhautmelanome im Kopf-HalsBereich werden in der Regel bei älteren Menschen beobachtet.
Der Häufigkeitsgipfel liegt im 5. und 7. Lebensjahrzehnt [4,14]. Die deutliche Häufigkeit der Schleimhautmelanome in einem höheren Lebensalter gilt auch für die Tumormanifestation in den Nasen- und Nasennebenhöhlen. Bei den von Holdcraft und Gallagher ausgewerteten Erkrankungsfällen waren zum Zeitpunkt der Diagnose nur $8 \%$ der betroffenen Patienten jünger als 40 Jahre, während $63 \%$ zwischen 50 und 79 Jahre alt waren [12]. Die Angaben zur Geschlechtsverteilung der Schleimhautmelanome im Kopf-Hals-Bereich zeigen von Ausnahmen abgesehen ein deutlich häufigeres Vorkommen bei männlichen Patienten $[4,13,14]$. Die gleiche Einschätzung gilt wiederum auch für die spezielle Lokalisation des Tumors im Bereich der Nasen- und Nasennebenhöhlen. Bei den von Holdcraft und Gallagher bzw. der Arbeitsgruppe um Freedman ausgewerteten Daten waren 59-62\% männliche und $38-41 \%$ weibliche Patienten gewesen $[11,12]$.

Die klinische Symptomatik der Schleimhautmelanome zeigt sich üblicherweise erst bei einem fortgeschrittenen Tumorwachstum. Im Bereich der Nasenhöhlen sind Blutungen und eine Behinderung der Nasenatmung durch Verlegung der nasalen Atemwege die häufigsten Symptome. Auch Tubenbelüftungsstörungen mit Einschränkung der Gehörfunktion und die Epiphora, das Tränenträufeln durch Verlegung des Ductus nasolacrimalis, zählen zur weiteren Symptomatik [11]. Bei einem Schleimhautmelanom im Bereich der Nasennebenhöhlen stehen dumpfe, druckartige Schmerzen im Vordergrund, die bisweilen als Zahnschmerzen fehlgedeutet werden [15]. Schwellungen und Parästhesien der Haut über den betroffenen Nasennebenhöhlen gelten hier ebenfalls als typische Symptome [11,12]. Die klinische Morphologie der Schleimhautmelanome ist in den meisten Fällen durch singulär-knotige oder polypoide Strukturen gekennzeichnet. Neben einer kräftigen Pigmentierung finden sich auch amelanotische Formen. Oberflächliche Nekrosen mit hämorrhagischen Krusten und eine deutliche Vulnerabilität mit ausgeprägter Neigung zu Blutungen sind weitere morphologische Eigenschaften $[12,16,17]$. Bei der Differenzialdiagnose der Schleimhautmelanome der Nasen- und Nasennebenhöhlen sind bei pigmentierten Formen Hämangiome, Kaposi-Sarkome und pigmentierte Metastasen maligner Melanome anderer Lokalisationen zu berücksichtigen. Amelanotische, polypöse Schleimhautmelanome müssen hingegen von Schleimhautpolypen, Sarkomen oder Metastasen anderer Malignome abgegrenzt werden $[14,15,18]$.

Die Prognose maligner Schleimhautmelanome im Kopf-Hals-Bereich wird überwiegend als sehr ungünstig angesehen. Allerdings finden sich in der Literatur neben 5-Jahres-Überlebensraten von 6,10 und $17 \%$ auch Raten von $45-48 \%$ [14,19-22]. Diese teilweise deutlich voneinander abweichenden Ergebnisse einzelner Untersuchungen sind unter anderem darauf zurückzuführen, dass international anerkannte, vergleichbare Einteilungskriterien der lokalen Tumorausdehnung nicht vorhanden sind. Die vereinzelt auch bei Schleimhautmelanomen geforderten Bestimmungen der Tumordicke nach Breslow oder des prognostischen Index nach Schmoeckel haben sich als Routineverfahren in der Praxis ebenfalls nicht durchgesetzt [23]. Somit lässt sich die Prognose der Schleimhautmelanome nicht auf der Grundlage einer histopathologischen Datensammlung einschätzen, obwohl offensichtlich ein Zusammenhang zwischen der Tumordicke und der Prognose besteht. Bei oralen Schleimhautmelanomen fanden 
Batsakis et al. eine schlechtere Prognose bei einem vertikalen Tumordurchmesser von mehr als $0,5 \mathrm{~mm}$ im Vergleich zu dünneren Schleimhautmelanomen [24]. Als weitere ungünstige Prognosekriterien gelten Lymphknotenmetastasen zum Zeitpunkt der Diagnose eines Schleimhautmelanoms, die Tumormanifestation im Bereich der Nasennebenhöhlen und amelanotische Tumorformen $[4,11,13,14]$. Vor dem Hintergrund der schlechten Prognose sind unterschiedliche Therapieverfahren angewendet worden. Einigkeit besteht darin, dass eine möglichst weiträumige Exzision des Tumors die einzige kurative Behandlungsform darstellt $[21,25]$. Ist eine komplette Resektion nicht mehr möglich, besteht die Indikation zur postoperativen Radiotherapie unter Einbeziehung der ersten Lymphknotenstation $[21,26]$. Darüber hinaus wurde vereinzelt auch über eine tendenzielle Verbesserung der Prognose berichtet, wenn neben der chirurgischen Sanierung eine adjuvante Radiotherapie durchgeführt worden ist $[9,11,27]$. Die Indikation zur prophylaktischen neck dissection wird heute verneint, während eine radikale Lymphknotenausräumung bei nachgewiesener lymphogener Metastasierung die Überlebenszeit zu verbessern scheint $[21,28]$. Die Chemotherapie, zumeist mit Dacarbazin durchgeführt, zeigt weder bei adjuvanter noch bei therapeutischer Indikation eine Verbesserung der Überlebenszeit [26].

Als Fazit auch der hier vorgestellten Kasuistik bleibt abschließend festzustellen, dass die selten vorkommenden und häufig erst in einem fortgeschrittenen Stadium diagnostizierten Schleimhautmelanome der Nasen- und Nasennebenhöhlen eine insgesamt schlechte Prognose aufweisen. Mit Ausnahme einer frühzeitigen Operation haben die vorhandenen Behandlungsmöglichkeiten zu keiner sicher erkennbaren Verbesserung der Prognose geführt.

\section{Literatur}

${ }^{1}$ Weidner F, Tonak J. Das maligne Melanom der Haut. 1. Aufl. Erlangen: perimed, 1981

2 Garbe C. Zentralregister Malignes Melanom der Deutschen Dermatologischen Gesellschaft, Stand Oktober 2005

${ }^{3}$ Ballantyne AJ. Malignant melanoma of the skin of the head and neck. Am J Surg 1970; 120: 425-431

${ }^{4}$ Conley J, Pack GT. Melanoma of the mucous membranes of the head und neck. Arch Otolaryngol 1974; 99: 315-319

${ }^{5}$ Moore ES, Martin H. Melanoma of the upper respiratory tract and oral cavity. Cancer 1955; 8: 1167-1176

${ }^{6}$ Pantazopoulos PE. Primary malignant melanoma of the nasal fossa. Ann Otol Rhinol Laryngol 1965; 74: 604-610
7 Shah JP, Huvos AG, Strong EW. Mucosal melanomas of the head and neck. Am J Surg 1977; 134: $531-535$

${ }^{8}$ Carducci A, Mazzilli G. Su un caso di melanoma maligno della tonsilla palatina. Otorhinolaring (Ital) 1965; 34: 507-511

${ }^{9}$ Folz BJ, Werner JA. Primärdiagnostik und Therapie der Schleimhautmelanome des aerodigestiven Traktes. JDDG 2005; 3: 830

${ }^{10}$ Harwood AR, Cummings BJ. Radiotherapy for mucosal melanomas. Int J Rad Onco Bio Phys 1982; 8: 1121 - 1126

${ }^{11}$ Freedman HM, DeSanto LW, Devine KD et al. Malignant melanoma of the nasal cavity und paranasal sinuses. Arch Otolaryngol 1973; 97: $322-325$

12 Holdcraft J, Callagher JC. Malignant melanoma of the nasal and paranasal sinus mucosa. Ann Otol Rhinol Laryngol 1969; 78: 5-20

${ }^{13}$ Nandapalan V, Roland NJ, Helliwell TR et al. Mucosal melanoma of the head and neck. Clin Otolaryngol 1998; 23: 107-116

${ }^{14}$ Enroth CM, Lundberg C. Mucosal malignant melanomas of the head and neck. Acta Otolaryngol 1975; 80: 452-458

${ }^{15}$ Wustrow F. Bösartige Tumoren der Nase und ihrer Nebenhöhlen. In: Berendes J, Link R, Zöllner F (Hrsg). Hals-Nasen-Ohren-Heilkunde in Praxis und Klinik. Stuttgart: Thieme, 1977

${ }^{16}$ Hormia M, Vuori EEJ. Mucosal melanoma of the head und neck. J Laryngol Otol 1969; 83: 349-359

${ }^{17}$ McLeod GR, Black RJ. Malignant melanomas cutaneous and mucosal. In: Paparella MM, Shumrick DA, Gluckmann W, Meyerhoff L (Hrsg). Otolaryngology. Philadelphia: Saunders, 1991

${ }^{18}$ Lookingball DP, Spangler N, Helm KF. Cutaneous metastases in patients with metastatic carcinoma: A retrospective study of 4020 patients. J Am Acad Dermatol 1993; 29: 228 - 236

${ }^{19}$ Lee SP, Shimizu KT, Tran LM et al. Mucosal melanoma of the head and neck: The impact of local control on survival. Laryngoscope 1994; 104: $121-126$

${ }^{20}$ Lund VJ. Malignant melanoma of the nasal and paranasal sinuses. Ear Nose Throat 1993; 72: 285-290

${ }^{21}$ Stern SJ, Guillamondegui O. Mucosal melanoma of the head and neck. Head Neck 1991; 13: 22 - 27

22 Ravid JM, Esteves JA. Malignant melanoma of the nose and paranasal sinuses and juvenile melanoma of the nose. Arch Otolaryngol (Chicago) 1960; 72: 431 - 444

${ }^{23}$ Nast-Kolb D, Landthaler M, Schweiberer L, Braun-Falco O. Anorectale maligne Melanome: Bericht von 7 eigenen Fällen und 374 Fällen aus der Literatur. Chirurg 1985; 56: 100-104

${ }^{24}$ Batsakis JS, Regezi JA, Solomon AR. The pathology of head and neck tumors. Head Neck Surg 1982; 4: 404-418

${ }^{25}$ Berthelsen A, Andersen AP, Jensen ST et al. Melanomas of the mucosa in the oral cavity and the upper respiratory passages. Cancer $1984 ; 54$ : 907-912

${ }^{26}$ Andersen LJ, Berthelsen A, Hansen HS. Malignant melanoma of the upper respiratory tract and the oral cavity. J Otolaryngol 1992; 21: 180185

${ }^{27}$ Kingdom TT, Kaplan MJ. Mucosal melanoma of the nasal cavity and paranasal sinuses. Head Neck 1995; 17: $184-189$

${ }^{28}$ O'Brien CJ, Petersen-Schäfer R, Ruark D et al. Radical, modified, and selective neck dissection for cutaneous malignant melanoma. Head Neck 1997; 19: 589 - 594 